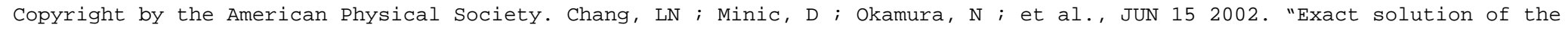

\title{
Exact solution of the harmonic oscillator in arbitrary dimensions with minimal length uncertainty relations
}

\author{
Lay Nam Chang, ${ }^{*}$ Djordje Minic, ${ }^{\dagger}$ Naotoshi Okamura, ${ }^{\ddagger}$ and Tatsu Takeuchi ${ }^{\S}$ \\ Institute for Particle Physics and Astrophysics, Physics Department, Virginia Tech, Blacksburg, Virginia 24061
}

(Received 20 November 2001; published 19 June 2002)

\begin{abstract}
We determine the energy eigenvalues and eigenfunctions of the harmonic oscillator where the coordinates and momenta are assumed to obey the modified commutation relations $\left[\hat{x}_{i}, \hat{p}_{j}\right]=i \hbar\left[\left(1+\beta \hat{p}^{2}\right) \delta_{i j}+\beta^{\prime} \hat{p}_{i} \hat{p}_{j}\right]$. These commutation relations are motivated by the fact that they lead to the minimal length uncertainty relations which appear in perturbative string theory. Our solutions illustrate how certain features of string theory may manifest themselves in simple quantum mechanical systems through the modification of the canonical commutation relations. We discuss whether such effects are observable in precision measurements on electrons trapped in strong magnetic fields.
\end{abstract}

DOI: 10.1103/PhysRevD.65.125027

PACS number(s): 03.65.Ge, 02.40.Gh

\section{INTRODUCTION}

In this paper, we derive the exact solution to the Schrödinger equation for the harmonic oscillator when the commutation relation between the position and momentum is modified from the canonical one to

$$
[\hat{x}, \hat{p}]=i \hbar\left(1+\beta \hat{p}^{2}\right) .
$$

This commutation relation leads to the uncertainty relation

$$
\Delta x \geqslant \frac{\hbar}{2}\left(\frac{1}{\Delta p}+\beta \Delta p\right),
$$

which implies the existence of a minimal length

$$
\Delta x_{\min }=\hbar \sqrt{\beta},
$$

below which the uncertainty in position, $\Delta x$, cannot be reduced $[1,2]$.

The minimal length uncertainty relation, Eq. (2), has appeared in the context of perturbative string theory [3] where it is a consequence of the fact that strings cannot probe distances below the string scale $\hbar \sqrt{\beta}$. Though the modified commutation relation, Eq. (1), has not yet been derived directly from string theory, the fact that it implies Eq. (2) suggests that it is one possible way in which certain features of string theory may manifest themselves in low energy quantum mechanical systems.

It should be noted, however, that Eq. (2) is not a ubiquitous prediction of string theory. Indeed, both in the realms of perturbative and nonperturbative string theory (where distances shorter than the string scale can be probed by D-branes [4]), another type of uncertainty relation involving both spatial and time coordinates has been found to hold [5]. The distinction (and relation) between the minimal length

\footnotetext{
*Electronic address: laynam@vt.edu

†Electronic address: dminic@vt.edu

¥Electronic address: nokamura@vt.edu

${ }^{\S}$ Electronic address: takeuchi@vt.edu
}

uncertainty relation and the space-time uncertainty relation has been elucidated by Yoneya [6].

Nonetheless, Eq. (2) does embody an intriguing UV/IR relation : when $\Delta p$ is large (UV), $\Delta x$ is proportional to $\Delta p$ and therefore is also large (IR). This type of UV/IR relation has appeared in several other contexts: the AdS/CFT correspondence [7], noncommutative field theory [8], and more recently in attempts at understanding quantum gravity in asymptotically de Sitter spaces $[9,10]$.

It has been argued that the kind of UV/IR "bootstrap" described by Eq. (2) is necessary to understand observable implications of short distance physics on inflationary cosmology [11]. Likewise, Banks has argued that some kind of UV/IR relation should be at the core of the cosmological constant problem [12] as well as its relation to the problem of supersymmetry breaking. Therefore, both Eq. (2) and the underlying Eq. (1) are well motivated by a variety of applications, including the cosmological constant problem which we will discuss is a subsequent paper [13].

Furthermore, the UV/IR relation represented by Eq. (2) suggests that certain "stringy" short distance (UV) effects may manifest themselves at longer distances (IR), lending additional justification to our analysis of the nonrelativistic harmonic oscillator.

The problem of solving for the energy eigenvalues and eigenstates of the harmonic oscillator with the minimal length uncertainty relation has been studied previously by Kempf et al. in Refs. [1,2]. However, the exact result had been obtained only for the 1-dimensional case. We present here the exact solution for the general $D$-dimensional isotropic harmonic oscillator.

\section{THE HARMONIC OSCILLATOR IN 1 DIMENSION}

We represent the position and momentum operators obeying Eq. (1) in momentum space by

$$
\begin{aligned}
& \hat{x}=i \hbar\left[\left(1+\beta p^{2}\right) \frac{\partial}{\partial p}+\gamma p\right], \\
& \hat{p}=p .
\end{aligned}
$$


The choice of the constant $\gamma$ determines the weight function in the definition of the inner product:

$$
\langle f \mid g\rangle=\int \frac{d p}{\left(1+\beta p^{2}\right)^{1-\alpha}} f^{*}(p) g(p),
$$

where

$$
\alpha=\frac{\gamma}{\beta} .
$$

This definition ensures the hermiticity of $\hat{x}$. In the following, we will keep $\gamma$ arbitrary, though eventually we will find that the energy levels in fact do not depend on its value.

The Schrödinger equation for the harmonic oscillator with Hamiltonian

$$
\hat{H}=\frac{1}{2} \mu \omega^{2} \hat{x}^{2}+\frac{1}{2 \mu} \hat{p}^{2},
$$

is given by

$$
\begin{gathered}
{\left[-\mu \hbar \omega\left\{\left(\left(1+\beta p^{2}\right) \frac{\partial}{\partial p}\right)^{2}+2 \gamma p\left(\left(1+\beta p^{2}\right) \frac{\partial}{\partial p}\right)\right.\right.} \\
\left.\left.+\gamma(\beta+\gamma) p^{2}+\gamma\right\}+\frac{1}{\mu \hbar \omega} p^{2}\right] \Psi(p) \\
=\frac{2 E}{\hbar \omega} \Psi(p) .
\end{gathered}
$$

A change of variable from $p$ to

$$
\rho \equiv \frac{1}{\sqrt{\beta}} \tan ^{-1}(\sqrt{\beta} p)
$$

maps the region $-\infty<p<\infty$ to

$$
-\frac{\pi}{2 \sqrt{\beta}}<\rho<\frac{\pi}{2 \sqrt{\beta}},
$$

and casts Eq. (8) into the form:

$$
\begin{aligned}
& {\left[\mu \hbar \omega\left\{\frac{\partial^{2}}{\partial \rho^{2}}+\left(\frac{2 \gamma}{\sqrt{\beta}} \tan \sqrt{\beta} \rho\right) \frac{\partial}{\partial \rho}\right\}-\left\{\frac{1}{\mu \hbar \omega \beta}-\mu \hbar \omega \gamma\right.\right.} \\
& \left.\left.\times\left(1+\frac{\gamma}{\beta}\right)\right\} \tan ^{2} \sqrt{\beta} \rho+\left\{\frac{2 E}{\hbar \omega}+\mu \hbar \omega \gamma\right\}\right] \Psi(p)=0 .
\end{aligned}
$$

Defining dimensionless parameters by

$$
\xi \equiv \frac{\rho}{\sqrt{\mu \hbar \omega}}, \quad \kappa \equiv \sqrt{\mu \hbar \omega \beta}, \quad \delta \equiv \frac{\gamma}{\beta}, \quad \varepsilon \equiv \frac{2 E}{\hbar \omega},
$$

we obtain

$$
\begin{aligned}
& {\left[\frac{\partial^{2}}{\partial \xi^{2}}+2 \kappa \delta \frac{s}{c} \frac{\partial}{\partial \xi}-\left\{\frac{1}{\kappa^{2}}-\kappa^{2} \delta(1+\delta)\right\} \frac{s^{2}}{c^{2}}\right.} \\
& \left.+\left(\varepsilon+\kappa^{2} \delta\right)\right] \Psi(\xi)=0,
\end{aligned}
$$

where we use the shorthand notations

$$
c \equiv \cos \kappa \xi, \quad s \equiv \sin \kappa \xi .
$$

Let $\Psi(\xi)=c^{\lambda+\delta} f(s)$, where $\lambda$ is a constant to be determined. Then the equation for $f(s)$ is

$$
\begin{aligned}
& \left(1-s^{2}\right) f^{\prime \prime}-(2 \lambda+1) s f^{\prime}+\left[\left\{\frac{\varepsilon}{\kappa^{2}}-\lambda\right\}\right. \\
& +\left\{\lambda(\lambda-1)-\frac{1}{\kappa^{4}} \frac{s^{2}}{c^{2}}\right] f=0 .
\end{aligned}
$$

The variable is now $-1<s<1$. Note that $\delta=\gamma / \beta$ is eliminated from the equation. We fix $\lambda$ by requiring the coefficient of the tangent squared term to vanish:

$$
\lambda(\lambda-1)-\frac{1}{\kappa^{4}}=0 .
$$

The wave function should be nonsingular at $c=0$, which implies

$$
\lambda=\frac{1}{2}+\sqrt{\frac{1}{4}+\frac{1}{\kappa^{4}}} .
$$

This simplifies Eq. (15) to

$$
\left(1-s^{2}\right) f^{\prime \prime}-(2 \lambda+1) s f^{\prime}+\left(\frac{\varepsilon}{\kappa^{2}}-\lambda\right) f=0 .
$$

Similarly, $f(s)$ should be nonsingular at $s= \pm 1$. Thus we require a polynomial solution to Eq. (18). This requirement imposes the following condition on the coefficient of $f$ :

$$
\frac{\varepsilon}{\kappa^{2}}-\lambda=n(n+2 \lambda),
$$

where $n$ is a non-negative integer [15]. Equation (18) becomes

$$
\left(1-s^{2}\right) f^{\prime \prime}-(2 \lambda+1) s f^{\prime}+n(n+2 \lambda) f=0,
$$

the solution of which is given by the Gegenbauer polynomial:

$$
f(s)=C_{n}^{\lambda}(s) .
$$

The energy eigenvalues follow from the condition Eq. (19):

$$
\begin{aligned}
\varepsilon_{n} & =\kappa^{2}\left[n^{2}+(2 n+1) \lambda\right] \\
& =\kappa^{2}\left(n^{2}+n+\frac{1}{2}\right)+(2 n+1) \sqrt{1+\frac{\kappa^{4}}{4}},
\end{aligned}
$$

or more explicitly, 


$$
\begin{aligned}
E_{n}= & \hbar \omega\left[\left(n+\frac{1}{2}\right) \sqrt{1+\frac{\beta^{2} \mu^{2} \hbar^{2} \omega^{2}}{4}}\right. \\
& \left.+\left(n^{2}+n+\frac{1}{2}\right) \frac{\beta \mu \hbar \omega}{2}\right] .
\end{aligned}
$$

This result agrees with Kempf [1]. The normalized energy eigenfunctions are

$$
\Psi_{n}(p)=2^{\lambda} \Gamma(\lambda) \sqrt{\frac{n !(n+\lambda) \sqrt{\beta}}{2 \pi \Gamma(n+2 \lambda)}} c^{\lambda+\delta} C_{n}^{\lambda}(s),
$$

where

$$
\begin{aligned}
& c=\cos \sqrt{\beta} \rho=\frac{1}{\sqrt{1+\beta p^{2}}}, \\
& s=\sin \sqrt{\beta} \rho=\frac{\sqrt{\beta} p}{\sqrt{1+\beta p^{2}}} .
\end{aligned}
$$

\section{THE HARMONIC OSCILLATOR IN $D$ DIMENSIONS}

In more than 1 dimension, the modified commutation relation can be generalized to the tensorial form:

$$
\left[\hat{x}_{i}, \hat{p}_{j}\right]=i \hbar\left(\delta_{i j}+\beta \hat{p}^{2} \delta_{i j}+\beta^{\prime} \hat{p}_{i} \hat{p}_{j}\right)
$$

If the components of the momentum $\hat{p}_{i}$ are assumed to commute with each other,

$$
\left[\hat{p}_{i}, \hat{p}_{j}\right]=0
$$

then the commutation relations among the coordinates $\hat{x}_{i}$ are almost uniquely determined by the Jacobi identity (up to possible extensions) as [1,2]

$$
\left[\hat{x}_{i}, \hat{x}_{j}\right]=i \hbar \frac{\left(2 \beta-\beta^{\prime}\right)+\left(2 \beta+\beta^{\prime}\right) \beta \hat{p}^{2}}{\left(1+\beta \hat{p}^{2}\right)}\left(\hat{p}_{i} \hat{x}_{j}-\hat{p}_{j} \hat{x}_{i}\right) .
$$

These operators are realized in momentum space as

$$
\begin{aligned}
& \hat{x}_{i}=i \hbar\left[\left(1+\beta p^{2}\right) \frac{\partial}{\partial p_{i}}+\beta^{\prime} p_{i} p_{j} \frac{\partial}{\partial p_{j}}+\gamma p_{i}\right], \\
& \hat{p}_{i}=p_{i} .
\end{aligned}
$$

The arbitrary constant $\gamma$ in the representation of $\hat{x}_{i}$ does not affect the commutation relations among the $\hat{x}_{i}$, s. Again, its choice determines the weight function in the definition of the inner product:

$$
\langle f \mid g\rangle=\int \frac{d^{D} \mathbf{p}}{\left[1+\left(\beta+\beta^{\prime}\right) p^{2}\right]^{1-\alpha}} f^{*}(\mathbf{p}) g(\mathbf{p}),
$$

where

$$
\alpha=\frac{\gamma-\beta^{\prime}\left(\frac{D-1}{2}\right)}{\left(\beta+\beta^{\prime}\right)} .
$$

Note that when $\beta^{\prime}=0$, this expression reduces to that of the $1 \mathrm{D}$ case, Eq. (6). Reference [2] uses

$$
\gamma=\beta+\beta^{\prime}\left(\frac{D+1}{2}\right)
$$

in which case the weight function is constant. We will keep $\gamma$ arbitrary in our calculations. As in the 1D case, we will find that the energy eigenvalues do not depend on $\gamma$.

Since the $D$-dimensional harmonic oscillator

$$
\hat{H}=\frac{1}{2} \mu \omega^{2} \hat{\mathbf{x}}^{2}+\frac{1}{2 \mu} \hat{\mathbf{p}}^{2}
$$

is rotationally symmetric, we can assume that the momentum space energy eigenfunctions expressed in terms of the radial momenta can be written as a product of spherical harmonics and a radial wave function:

$$
\Psi_{D}(\mathbf{p})=Y_{l_{(D-1)} \cdots l_{2} l_{1}}(\Omega) R(p)
$$

In $2 \mathrm{D}$ and $3 \mathrm{D}$, we have

$$
\begin{aligned}
& \Psi_{2}(\mathbf{p})=\frac{1}{\sqrt{2 \pi}} e^{-i m \phi} R(p), \\
& \Psi_{3}(\mathbf{p})=Y_{l m}(\theta, \phi) R(p) .
\end{aligned}
$$

Equation (34) allows us to make the replacements

$$
\begin{aligned}
& \sum_{i=1}^{N} \frac{\partial^{2}}{\partial p_{i}^{2}}=\frac{\partial^{2}}{\partial p^{2}}+\frac{D-1}{p} \frac{\partial}{\partial p}-\frac{L^{2}}{p^{2}}, \\
& \sum_{i=1}^{N} p_{i} \frac{\partial}{\partial p_{i}}=p \frac{\partial}{\partial p},
\end{aligned}
$$

where [14]

$$
L^{2}=l(l+D-2), \quad l=0,1,2, \cdots .
$$

$(l=|m|$ for $D=2$.

We therefore find, not unexpectedly, that the Schrödinger equation for the $D$-dimensional harmonic oscillator can be reduced to the 1-dimensional problem for the radial wave function $R(p)$. The equation for $R(p)$ is: 


$$
\begin{aligned}
& -\mu \hbar \omega\left[\left\{\left[1+\left(\beta+\beta^{\prime}\right) p^{2}\right] \frac{\partial}{\partial p}\right\}^{2}+\left\{\frac{D-1}{p}+[(D-1) \beta+2 \gamma] p\right\}\left\{\left[1+\left(\beta+\beta^{\prime}\right) p^{2}\right] \frac{\partial}{\partial p}\right\}\right. \\
& \left.-\frac{L^{2}}{p^{2}}+\left(\gamma D-2 \beta L^{2}\right)+\left\{\gamma\left(\beta D+\beta^{\prime}+\gamma\right)-\beta^{2} L^{2}\right\} p^{2}\right] R(p)+\frac{1}{\mu \hbar \omega} p^{2} R(p)=\frac{2 E}{\hbar \omega} R(p) .
\end{aligned}
$$

Introducing the change of variable

$$
\rho \equiv \frac{1}{\sqrt{\beta+\beta^{\prime}}} \tan ^{-1} \sqrt{\beta+\beta^{\prime}} p
$$

maps the region $0<p<\infty$ to

$$
0<\rho<\frac{\pi}{2 \sqrt{\beta+\beta^{\prime}}},
$$

and renders the equation into the following form:

$$
\begin{gathered}
{\left[-\mu \hbar \omega\left\{\frac{\partial^{2}}{\partial \rho^{2}}+\left(\frac{(D-1) \sqrt{\beta+\beta^{\prime}}}{\tan \sqrt{\beta+\beta^{\prime}} \rho}+\frac{[(D-1) \beta+2 \gamma]}{\sqrt{\beta+\beta^{\prime}}} \tan \sqrt{\beta+\beta^{\prime}} \rho\right) \frac{\partial}{\partial \rho}-\frac{L^{2}\left(\beta+\beta^{\prime}\right)}{\tan ^{2} \sqrt{\beta+\beta^{\prime}} \rho}+\left(\gamma D-2 \beta L^{2}\right)\right.\right.} \\
\left.\left.+\frac{\left[\gamma\left(\beta D+\beta^{\prime}+\gamma\right)\right]-\beta^{2} L^{2}}{\left(\beta+\beta^{\prime}\right)} \tan ^{2} \sqrt{\beta+\beta^{\prime}} \rho\right\}+\frac{1}{\mu \hbar \omega\left(\beta+\beta^{\prime}\right)} \tan ^{2} \sqrt{\beta+\beta^{\prime}} \rho\right] R(\rho)=\frac{2 E}{\hbar \omega} R(\rho) .
\end{gathered}
$$

Defining the dimensionless parameters

$$
\xi \equiv \frac{\rho}{\sqrt{\mu \hbar \omega}}, \quad \kappa \equiv \sqrt{\mu \hbar \omega\left(\beta+\beta^{\prime}\right)}, \quad \eta \equiv \frac{\beta}{\beta+\beta^{\prime}}, \quad \delta \equiv \frac{\gamma}{\beta+\beta^{\prime}}, \quad \varepsilon \equiv \frac{2 E}{\hbar \omega},
$$

we obtain

$$
\begin{aligned}
& \frac{1}{\kappa^{2}} \frac{\partial^{2} R}{\partial \xi^{2}}+\left[(D-1) \frac{c}{s}+\{(D-1) \eta+2 \delta\} \frac{s}{c}\right] \frac{1}{\kappa} \frac{\partial R}{\partial \xi} \\
& +\left[\left\{\frac{\varepsilon}{\kappa^{2}}-(2 \eta-1) L^{2}+\delta D\right\}-\frac{L^{2}}{s^{2}}+\left\{\delta(D-1) \eta+\delta(1+\delta)-\eta^{2} L^{2}-\frac{1}{\kappa^{4}}\right\} \frac{s^{2}}{c^{2}}\right] R=0,
\end{aligned}
$$

where we use the shorthand notation

$$
c=\cos \kappa \xi, \quad s=\sin \kappa \xi
$$

as before. Let $R(\xi)=c^{\lambda+\delta} f(s)$. The equation for $f(s)$ is

$$
\begin{aligned}
& \left(1-s^{2}\right) f^{\prime \prime}+\left[-\{2 \lambda+1+(D-1)(1-\eta)\} s+\frac{D-1}{s}\right] f^{\prime} \\
& +\left[\left\{\frac{\varepsilon}{\kappa^{2}}-(2 \eta-1) L^{2}-\lambda D\right\}-\frac{L^{2}}{s^{2}}+\left\{\lambda^{2}-\lambda[1+(D-1) \eta]-\eta^{2} L^{2}-\frac{1}{\kappa^{4}}\right\} \frac{s^{2}}{c^{2}}\right] f=0 .
\end{aligned}
$$


Note that as in the $1 \mathrm{D}$ case, $\delta$ is eliminated. We choose $\lambda$ to cancel the tangent squared term:

$$
\lambda^{2}-\lambda[1+(D-1) \eta]-\eta^{2} L^{2}-\frac{1}{\kappa^{4}}=0
$$

Taking the positive root, we obtain

$$
\lambda=\frac{1+(D-1) \eta}{2}+\sqrt{\frac{\{1+(D-1) \eta\}^{2}}{4}+\eta^{2} L^{2}+\frac{1}{\kappa^{4}}}
$$

The equation for $f(s)$ then simplifies to

$$
\begin{aligned}
& \left(1-s^{2}\right) f^{\prime \prime}+\left[-\{2 \lambda+1+(D-1)(1-\eta)\} s+\frac{D-1}{s}\right] f^{\prime} \\
& +\left[\left\{\frac{\varepsilon}{\kappa^{2}}-\lambda D-(2 \eta-1) L^{2}\right\}-\frac{L^{2}}{s^{2}}\right] f=0
\end{aligned}
$$

Next, let $f(s)=s^{l} g(s)$. This substitution eliminates the centrifugal barrier term and gives the equation for $g(s)$ :

$$
\left(1-s^{2}\right) g^{\prime \prime}+\left[-\{2 \lambda+2 l+1+(D-1)(1-\eta)\} s+\frac{2 l+D-1}{s}\right] g^{\prime}+\left[\frac{\varepsilon}{\kappa^{2}}-2 \eta L^{2}-(2 l+D) \lambda+l\{(D-1) \eta-1\}\right] g=0 .
$$

Another change of variable

$$
z=2 s^{2}-1
$$

maps the interval $0<s<1$ to $-1<z<1$ and leads to

$$
\left(1-z^{2}\right) \frac{d^{2} g}{d z^{2}}+[(b-a)-(a+b+2) z] \frac{d g}{d z}+\frac{1}{4}\left[\frac{\varepsilon}{\kappa^{2}}-2 \eta L^{2}-(2 l+D) \lambda+l\{(D-1) \eta-1\}\right] g=0,
$$

where

$$
\begin{aligned}
a & =\lambda-\frac{1+(D-1) \eta}{2} \\
& =\sqrt{\frac{\{1+(D-1) \eta\}^{2}}{4}+\eta^{2} L^{2}+\frac{1}{\kappa^{4}}}, \\
b & =\frac{D}{2}+l-1 .
\end{aligned}
$$

Once again, we need a polynomial solution to $g(z)$ to keep the wave function regular at $z= \pm 1$. The condition one must impose is

$$
\begin{aligned}
& \frac{1}{4}\left[\frac{\varepsilon}{\kappa^{2}}-2 \eta L^{2}-(2 l+D) \lambda+l\{(D-1) \eta-1\}\right] \\
& \quad=n^{\prime}\left(n^{\prime}+a+b+1\right)
\end{aligned}
$$

where $n^{\prime}$ is a non-negative integer [15]. This casts Eq. (51) into the form

$$
\begin{aligned}
& \left(1-z^{2}\right) \frac{d^{2} g}{d z^{2}}+[(b-a)-(a+b+2) z] \frac{d g}{d z} \\
& +n^{\prime}\left(n^{\prime}+a+b+1\right) g=0,
\end{aligned}
$$

$$
\begin{aligned}
\frac{\varepsilon}{\kappa^{2}} & =2\left[\left(2 n^{\prime}+l\right)+\frac{D}{2}\right] \lambda+\left[4 n^{\prime 2}+2 n^{\prime}(1-\eta)(D-1)+4 n^{\prime} l+2 \eta L^{2}+\{\eta(D-1)-1\} l\right] \\
& =2\left(n+\frac{D}{2}\right) \lambda+\left[n^{2}+n(1-\eta)(D-1)+(2 \eta-1) L^{2}\right] \\
& =2\left(n+\frac{D}{2}\right) \sqrt{\frac{\{1+(D-1) \eta\}^{2}}{4}+\eta^{2} L^{2}+\frac{1}{\kappa^{4}}}+\left[n^{2}+n D+\frac{D^{2}}{2} \eta+\frac{D}{2}(1-\eta)+(2 \eta-1) L^{2}\right],
\end{aligned}
$$

The energy eigenvalues are given by 
where in the second line we have made the replacement $2 n^{\prime}+l=n$. The final expression is:

$$
E_{n l}=\hbar \omega\left[\left(n+\frac{D}{2}\right) \sqrt{1+\left\{\beta^{2} L^{2}+\frac{\left(D \beta+\beta^{\prime}\right)^{2}}{4}\right\} \mu^{2} \hbar^{2} \omega^{2}}+\left\{\left(\beta+\beta^{\prime}\right)\left(n+\frac{D}{2}\right)^{2}+\left(\beta-\beta^{\prime}\right)\left(L^{2}+\frac{D^{2}}{4}\right)+\beta^{\prime} \frac{D}{2}\right\} \frac{\mu \hbar \omega}{2}\right] .
$$

This equation represents the main result of our paper. The 1D result can be reproduced from this expression by setting $D$ $=1, L^{2}=0$, and $\beta^{\prime}=0$. The normalized energy eigenfunctions are

$$
R_{n l}(p)=\sqrt{\frac{2\left(2 n^{\prime}+a+b+1\right) n^{\prime} ! \Gamma\left(n^{\prime}+a+b+1\right)}{\Gamma\left(n^{\prime}+a+1\right) \Gamma\left(n^{\prime}+b+1\right)}}\left(\beta+\beta^{\prime}\right)^{D / 4} c^{\lambda+\delta} s^{l} P_{n^{\prime}}^{(a, b)}(z),
$$

where $n^{\prime}=(n-l) / 2$, and

$$
\begin{aligned}
& c=\cos \sqrt{\beta+\beta^{\prime}} \rho=\frac{1}{\sqrt{1+\left(\beta+\beta^{\prime}\right) p^{2}}}, \\
& s=\sin \sqrt{\beta+\beta^{\prime}} \rho=\frac{\sqrt{\beta+\beta^{\prime}} p}{\sqrt{1+\left(\beta+\beta^{\prime}\right) p^{2}}}, \\
& z=2 s^{2}-1=\frac{\left(\beta+\beta^{\prime}\right) p^{2}-1}{\left(\beta+\beta^{\prime}\right) p^{2}+1} .
\end{aligned}
$$

\section{COMPARISON WITH PREVIOUS RESULTS}

Kempf [2] has calculated the energy eigenvalues of the 2D and 3D harmonic oscillators to linear order in $\beta$ and $\beta^{\prime}$. From the exact expression Eq. (57), we can easily identify the terms to that order to be:

$$
E_{n l} \approx \hbar \omega\left[\left(n+\frac{D}{2}\right)+\frac{1}{2}\left\{\left(k^{2}+k^{\prime 2}\right)\left(n+\frac{D}{2}\right)^{2}+\left(k^{2}-k^{\prime 2}\right)\left(L^{2}+\frac{D^{2}}{4}\right)+k^{\prime 2} \frac{D}{2}\right\}\right] .
$$

For ease of comparison, we have introduced the notation

$$
k^{2}=\beta \mu \hbar \omega, \quad k^{\prime 2}=\beta^{\prime} \mu \hbar \omega .
$$

For the $D=2$ and $D=3$ cases, the explicit expressions are

$$
\begin{aligned}
& E_{2 D} \approx \hbar \omega\left[(n+1)+\frac{1}{2}\left\{\left(k^{2}+k^{\prime 2}\right)(n+1)^{2}+\left(k^{2}-k^{\prime 2}\right)\left(l^{2}+1\right)+k^{\prime 2}\right\}\right], \\
& E_{3 D} \approx \hbar \omega\left[\left(n+\frac{3}{2}\right)+\frac{1}{2}\left\{\left(k^{2}+k^{\prime 2}\right)\left(n+\frac{3}{2}\right)^{2}+\left(k^{2}-k^{\prime 2}\right)\left(l(l+1)+\frac{9}{4}\right)+k^{\prime 2} \frac{3}{2}\right\}\right] .
\end{aligned}
$$

We define the parameter $s$ by

$$
s \equiv n^{\prime}+1=\frac{n-l}{2}+1
$$

which takes values from 1 to $[(n+2) / 2]$ for fixed $n$. Then

$$
\begin{aligned}
& E_{2 D} \approx \hbar \omega\left[(n+1)+k^{2}\left(n^{2}+3 n+3\right)-k^{\prime 2}\left(n+\frac{3}{2}\right)-2\left(k^{2}-k^{\prime 2}\right) s(n+2-s)\right], \\
& E_{3 D} \approx \hbar \omega\left[\left(n+\frac{3}{2}\right)+k^{2}\left(n^{2}+4 n+\frac{21}{4}\right)-k^{\prime 2}\left(n+\frac{9}{4}\right)-\left(k^{2}-k^{\prime 2}\right) s(2 n+5-2 s)\right],
\end{aligned}
$$

which agree with Ref. [2]. See also Ref. [16]. 


\section{ELECTRONS IN A PENNING TRAP}

The $n^{2}$ dependence of the harmonic oscillator energy levels, Eq. (57), would be an unmistakeable signature of the modified commutation relations Eq. (1) or Eq. (26). Even if $\beta$ and $\beta^{\prime}$ were small, the deviation from the usual $n$ dependence should be manifest for sufficiently large $n$.

The cyclotron motion of an electron in a Penning trap [17] is effectively a one-dimensional harmonic oscillator. By looking for shifts in its energy levels, we may be able to place a constraint on $\beta$. To leading order in $\beta$ and $n$, the deviation of the harmonic oscillator energy levels from the canonical $\hbar \omega\left(n+\frac{1}{2}\right)$ is given by

$$
\frac{\Delta E_{n}}{\hbar \omega}=\left(\frac{\beta m \hbar \omega}{2}\right) n^{2},
$$

which grows quickly with $n$. Note that the combination of parameters that is constained by a measurement of $\Delta E_{n} / \hbar \omega$ is $\beta m \hbar \omega$. The cyclotron frequency of an electron trapped in a magnetic field of strength $B$ is (in SI units)

$$
\omega_{c}=\frac{e B}{m_{e}} .
$$

Therefore,

$$
m_{e} \hbar \omega_{c}=(e \hbar) B=\left(1.7 \times 10^{-53} \mathrm{~kg}^{2} \mathrm{~m}^{2} / \mathrm{s}^{2} / \mathrm{T}\right) B,
$$

which is independent of the electron mass $m_{e}$. For a trapping field strength of $B=6 \mathrm{~T}$, we obtain

$$
m_{e} \hbar \omega_{c}=e \hbar B=\left(1.0 \times 10^{-52} \mathrm{~kg}^{2} \mathrm{~m}^{2} / \mathrm{s}^{2}\right) .
$$

Even though we anticipate that measuring the energy levels accurately for very large $n$ would be difficult, let us assume for the sake of argument that deviations as large as $\hbar \omega_{c}$ would be detectable. Then, the absence of such a deviation for the $n$th energy level would imply

$$
\left(\frac{\beta e \hbar B}{2}\right) n^{2}<1
$$

or

$$
\beta<\left(\frac{2}{e \hbar B}\right) \frac{1}{n^{2}}=\frac{\left(2.0 \times 10^{52} \mathrm{~m}^{2} / \mathrm{J}^{2} \mathrm{~s}^{2}\right)}{n^{2}}
$$

This translates into

$$
\hbar \sqrt{\beta}<\sqrt{\left(\frac{2 \hbar}{e B}\right) \frac{1}{n^{2}}}=\frac{\left(1.5 \times 10^{-8} \mathrm{~m}\right)}{n},
$$

as a limit for the minimal length, Eq. (3), and

$$
\begin{aligned}
\frac{1}{\sqrt{\beta}} & >\sqrt{\left(\frac{e \hbar B}{2}\right) n^{2}}=\left(7.1 \times 10^{-27} \mathrm{~kg} \mathrm{~m} / \mathrm{s}\right) n \\
& =(13 \mathrm{eV} / c) n,
\end{aligned}
$$

for the string momentum scale.

Aside from the technical question of whether one can measure the energy levels accurately for large $n$, we must require that $n$ satisfy

$$
\frac{n \hbar \omega_{c}}{m_{e} c^{2}} \ll 1
$$

for our electron to stay nonrelativistic. This leads to the constraint

$$
n \ll \frac{m_{e} c^{2}}{\hbar \omega_{c}}=\frac{\left(m_{e} c\right)^{2}}{(e \hbar) B} \approx 10^{9} .
$$

This condition also maintains the radius of the electron's cyclotron motion to be well within the geometry of the Penning trap. Therefore, the maximum value of $n$ that can be used to constrain $\beta$ would be $n \sim 10^{8}$ if we allow for a $10 \%$ relativistic correction. So the best limit on $\beta$ that can be achieved will be

$$
\hbar \sqrt{\beta}<10^{-16} \mathrm{~m}, \quad \frac{1}{\sqrt{\beta}}>1 \mathrm{GeV} / c .
$$

Obtaining a better limit would be difficult since improving the limit on $\sqrt{\beta}$ by an order of magnitude requires the improvement of the limit on $\beta$ by two orders of magnitude.

\section{DISCUSSION AND CONCLUSIONS}

We have obtained the exact energy eigenvalues and eigenstates of the harmonic oscillator when the coordinate and momentum operators satisfy the modified commutation relations Eq. (1) or Eq. (26).

The energy levels, Eqs. (23) and (57), grow as $n^{2}$ for large $n$. The reason for this $n^{2}$ behavior can be understood as follows: The change of variable from $p$ to $\rho$ in the $1 D$ problem changes the $p^{2}$ kinetic term into a $\tan ^{2} \sqrt{\beta} \rho$ potential which is bounded at $\rho= \pm \pi / 2 \sqrt{\beta}$. For higher dimensions, the effective potential is $\tan ^{2} \sqrt{\beta+\beta^{\prime}} \rho$ plus a centrifugal barrier $\cot ^{2} \sqrt{\beta+\beta^{\prime}} \rho$ which introduces a wall at $\rho=\pi / 2 \sqrt{\beta+\beta^{\prime}}$ in addition to the one at $\rho=0$. For higher energy eigenstates, the potentials are in essence square wells, leading to the $n^{2}$ dependence of the energy. Indeed, the energy eigenvalues of a spherically symmetric square well potential of radius $\pi / 2 \sqrt{\beta+\beta^{\prime}}$ are given approximately by

$$
E_{n} \approx \hbar \omega\left(\frac{\mu \hbar \omega}{2}\right)\left(\beta+\beta^{\prime}\right)\left(n+\frac{D+1}{2}\right)^{2} .
$$

The parameter $\gamma$, introduced in Eqs. (4) and (29) has no effect on the energy eigenvalues and only results in the wave functions acquiring an extra factor of

$$
\left[1+\left(\beta+\beta^{\prime}\right) p^{2}\right]^{-\delta / 2}
$$

which cancels the $\gamma$ dependence in the weight function of the inner product, Eqs. (5) and (30).

The original 


$$
\frac{(D+n-1) !}{(D-1) ! n !}
$$

fold degeneracy of the $n$th energy state is broken, leaving only the

$$
\frac{(D+l-1) !}{(D-1) ! l !}-\frac{(D+l-3) !}{(D-1) !(l-2) !}
$$

fold degeneracy for each value of $l$ due to rotational symmetry [14]. This loss of degeneracy can be interpreted as the breaking of self-supersymmetry of the harmonic oscillator [18]. The natural question arises whether an analogue exists on the level of field theory as a potentially new mechanism for supersymmetry breaking.

Potential constraints on $\beta$ that can be placed by measuring the energy levels of an electron trapped in a strong mag- netic field have been discussed. Even under optimistic assumptions, the constraints that can be imposed are weak.

In addition to affecting the energy levels of quantum mechanical systems, the modified commutation relations, Eqs. (1) and (26), may have other far reaching consequences. In subsequent papers, we will discuss their effects on the calculation of the cosmological constant [13], and the motion of macrosopic objects [19].

\section{ACKNOWLEDGMENTS}

We would like to thank Vijay Balasubramanian, Atsushi Higuchi, Asad Naqvi, Koenraad Schalm, Gary Shiu, Joseph Slawny, and Matthew Strassler for helpful discussions. This research is supported in part by the U.S. Department of Energy, Grant No. DE-FG05-92ER40709.
[1] A. Kempf, G. Mangano, and R.B. Mann, Phys. Rev. D 52, 1108 (1995).

[2] A. Kempf, J. Phys. A 30, 2093 (1997).

[3] D.J. Gross and P.F. Mende, Nucl. Phys. B303, 407 (1988); D.J. Gross and P.F. Mende, Phys. Lett. B 197, 129 (1987); D. Amati, M. Ciafaloni, and G. Veneziano, ibid. 216, 41 (1989); D. Amati, M. Ciafaloni, and G. Veneziano, Int. J. Mod. Phys. A 3, 1615 (1988); D. Amati, M. Ciafaloni, and G. Veneziano, Phys. Lett. B 197, 81 (1987); E. Witten, Phys. Today 49(4), 24 (1997).

[4] J. Polchinski, Phys. Rev. Lett. 75, 4724 (1995); M.R. Douglas, D. Kabat, P. Pouliot, and S.H. Shenker, Nucl. Phys. B485, 85 (1997).

[5] T. Yoneya, Int. J. Mod. Phys. A 16, 945 (2001); T. Yoneya, hep-th/9707002; M. Li and T. Yoneya, Phys. Rev. Lett. 78, 1219 (1997); M. Li and T. Yoneya, hep-th/9806240; H. Awata, M. Li, D. Minic, and T. Yoneya, J. High Energy Phys. 02, 013 (2001); D. Minic, Phys. Lett. B 442, 102 (1998).

[6] T. Yoneya, Prog. Theor. Phys. 103, 1081 (2000).

[7] L. Susskind and E. Witten, hep-th/9805114; A.W. Peet and J. Polchinski, Phys. Rev. D 59, 065011 (1999).

[8] M.R. Douglas and N.A. Nekrasov, Rev. Mod. Phys. 73, 977 (2002).

[9] C.M. Hull, J. High Energy Phys. 07, 021 (1998); C.M. Hull, ibid. 11, 017 (1998); V. Balasubramanian, P. Horava, and D. Minic, ibid. 05, 043 (2001); E. Witten, hep-th/0106109; A. Strominger, J. High Energy Phys. 10, 034 (2001).

[10] A. Strominger, J. High Energy Phys. 11, 049 (2001); V. Balasubramanian, J. de Boer, and D. Minic, Phys. Rev. D 65, 123508 (2002).

[11] A. Kempf, Phys. Rev. D 63, 083514 (2001); A. Kempf and J.C. Niemeyer, ibid. 64, 103501 (2001); R. Easther, B.R.
Greene, W.H. Kinney, and G. Shiu, ibid. 64, 103502 (2001); hep-th/0110226.

[12] T. Banks, Int. J. Mod. Phys. A 16, 910 (2001); hep-th/0007146; For other closely related attempts to understand the cosmological constant problem, consult, for example, T. Banks, hep-th/9601151; A.G. Cohen, D.B. Kaplan, and A.E. Nelson, Phys. Rev. Lett. 82, 4971 (1999); P. Horava and D. Minic, ibid. 85, 1610 (2000); N. Arkani-Hamed, S. Dimopoulos, N. Kaloper, and R. Sundrum, Phys. Lett. B 480, 193 (2000); S. Kachru, M. Schulz, and E. Silverstein, Phys. Rev. D 62, 045021 (2000).

[13] L.N. Chang, D. Minic, N. Okamura, and T. Takeuchi, Phys. Rev. D 65, 125028 (2002).

[14] A. Chodos and E. Myers, Ann. Phys. (N.Y.) 156, 412 (1984); A. Higuchi, J. Math. Phys. 28, 1553 (1987); N. IA. Vilenkin, Special Functions and the Theory of Group Representations (AMS, Providence, RI, 1968).

[15] I.S. Gradshteyn and I.M. Ryzhik, Table of Integrals, Series and Products 6th ed. (Academic, New York, 2000); M. Abramowitz and I.A. Stegun, Handbook of Mathematical Functions, with Formulas, Graphs, and Mathematical Tables (Dover, New York, 1974).

[16] F. Brau, J. Phys. A 32, 7691 (1999).

[17] L.S. Brown and G. Gabrielse, Rev. Mod. Phys. 58, 233 (1986); R.K. Mittleman, I.I. Ioannou, H.G. Dehmelt, and N. Russell, Phys. Rev. Lett. 83, 2116 (1999); H. Dehmelt, R. Mittleman, R.S. van Dyck, and P. Schwinberg, ibid. 83, 4694 (1999).

[18] F. Cooper and B. Freedman, Ann. Phys. (N.Y.) 146, 262 (1983).

[19] S. Benczik, L.N. Chang, D. Minic, N. Okamura, S. Rayyan, and T. Takeuchi, Phys. Rev. D (to be published), hep-th/0204049. 